\title{
Skin Conductance as Proxy for the Identification of Hydration Level in Human Body
}

\author{
A.Rizwan*;A.Zoha*;A. Alomainy†;N.A.Ali**;M.A.Imran*;Q.H.Abbasi* \\ *School of Engineering, University of Glasgow, Glasgow G12 8QQ, U.K \\ $\dagger$ School of Electronic Engineering and Computer Science, Queen Mary University of London, London \\ E1 4NS, U.K; **Faculty of Information Technology, United Arab Emirates University, UAE \\ Email:a.rizwan.1@ research.gla.ac.uk*; ahmed.zoha;muhammad.imran;qammer.abbasi\}@glasgow.ac.uk*; \\ a.alomainy@qmul.ac.uk†;najah@uaeu.ac.ae**;
}

\begin{abstract}
The skin dehydration level can be used to infer serious health conditions in patients since diseases like cardiovascular abnormality, diabetes and cancer symptoms do exhibit correlation with skin disorders. Therefore a systematic analysis of human skin hydration levels is critical for multiple health care applications. Motivated by this, in this study we proposed a unique approach of measuring body hydration levels against different body postures using skin conductance electrical activity. In this paper, we report the collection, processing and analysis techniques used in the analysis of skin conductance data. Subsequently in order to predict body hydration levels we employed state-of-the-art machine learning models using the skin conductance data and achieved $\mathbf{8 1 . 8 2 \%}$ and $73.91 \%$ recognition accuracy for the data of standing and sitting postures,respectively using KNN model.
\end{abstract}

Keywords: SCL, GSR, SCR, EDA, Hydration Level, Classification, Machine Learning, Bio-Sensors Data.

\section{INTRODUCTION}

Water is an essential nutrient for life and constitute the most of the body mass and volume. It is also the major constituent of the cell a building block of all the tissues and organs [1]. An appropriate hydration level is very important in human body for performing multiple body functions for example water being the major component of blood carries nutrients from food and oxygen from lungs to each part of the body. Dehydration can not only effect the performance of general body functions but it can also lead to serious diseases and even organ failures in its acute form. So, there is a need to monitor the body's hydration continuously as water is being lost waste exertion and replenished by the intake of food and fluid intake. It becomes more important in special medical conditions of illness, disease, or rigorous activity. In infants and elderly it is even more critical and sensitive to monitor the hydration level.

Unfortunately, currently, there exists no definitive gold standard for the quantitative measurement of hydration. However, researchers have explored the following parameters to quantitatively determine the body's state of hydration: plasma osmolality, total body water, lean body water, bio-electrical impedance, urine content and salivary flow rate [2]. Of these parameters, total body water (TBW) is currently widely accepted as a mean to determine the body's total hydration status [3]. Most of the solution available are invasive in nature like use of isotopic dilution for the measurement of total body water [4]. Whereas bio-electrical impedance analysis (BIA) is a well known noninvasive approach for determining body composition in terms of fat mass (FM), fat-free mass (FFM) and can also help to find total body water (TBW). BIA uses the resistance to the flow of electric current through body tissues that can be further used to estimate TBW [5]. But BIA is a complex, inconsistent and inaccurate method comparatively. Its main aim is to assess body composition and TBW is a by product of it. It is also difficult to convert the equipment involved into a wearable. Overall measuring hydration with a non-invasive methhod is a challenging task not only because of the scarcity of non-invasive resources that can sens it but also because it depends on multiple subject dependent factors like age, gender, height,BMI, fluid intakes, excretion patterns etc.

In research from solutions like BIA it is evident that there exists correlation between hydration level and body conductivity. Electric resistance (reciprocate of conductance) of human body varies between few ohms to thousands of ohms depending on body part and hydration level. More than $99 \%$ of the resistance to electric current flow to body's is at the skin [6]. So a method of measuring skin resistance or conductance can help to develop noninvasive wearable solution for hydration monitoring. Luckily there exist Electro-dermal activity (EDA) sensors which rely on the underlying technology of sensing the variations in the electrical properties of the skin. EDA is basically used for the study of sympathetic nervous system activities. Skin conductance or electro-dermal behaviour is very sensitive to electric signals generated by nervous system as the result of any internal or external stimuli. [7].

In this research we propose that EDA can be used as an indicator of different hydration levels as it measures the variation in the skin conductance which is also related to hydration level of the body. But one challenge is that EDA is very sensitive to internal and external stimuli for nervous system like happiness, fear, temperature,humidity etc. and can result into highly varying graph on short intervals. Whereas variation in hydration level is not an impulsive behaviour. This issue can be resolved by selecting appropriate interval of time and extracting features smartly for that interval such that instantaneous behaviour is set aside and long term behaviour is retained. So we have tried multiple window sizes for the 
time intervals, extracted multiple statistical features and used multiple machine learning algorithms. The goal is to identify the optimal window size, best combination of features and high accuracy algorithm with optimal tuning parameters to classify hyderation level on the basis of EDA data. To the best of our knowledge, it is for the first time that EDA is used to measure the hydration level.

Our major contributions here are:

1) Collection of electrical conductivity data for two distinct postures

2) Statistical analysis of the electrical conductivity of the skin and feature extraction

3) Application of the machine learning models for prediction of hydration levels.

The rest of the paper is organized as follows: Section II describes the data collected, Section III explains the whole process from data collection to model optimization along the tools used, results are presented in Sections IV and Section $\mathrm{V}$ concludes this paper.

\section{Sensing Data}

Data used in this research is collected from one subject for initial analysis and for the purpose of consistency as conductance can vary from individual to individual on the basis of factors apart from hydration level. Subject under study is a male of age round thirty, weight $70 \mathrm{KG}$ and height 57 ' with no conditions of edema or swear hypo-hydration. Data is collected over the period of one month but we have tried our best to keep the data collection environment consistent as EDA is very sensitive to internal and external parameters. Data is collected using BiTalino EDA sensor and processor [8]. For the collection of data pre-gelled active and passive electrodes are fixed to hypothenar sites on the palms of the non-dominant hand as it is recommended in [9] beside following other recommended precautions. Data is collected at a sampling rate of $1 \mathrm{MHz}$ and 16 bit resolution highest precision options provided by BiTalino EDA.

Data is collected in two states of the subject labelled as hydrated and dehydrated based on the condition whether the subject has drank water or not prior the reading. Subject is considered dehydrated when he has not drank water or consumed any other liquid or food for last 10 hours or more. Skin conductance measured within an hour of drinking water, consuming any liquid, is labelled as hydrated conductance level. Conductance is measured for five consecutive minutes at one time. Five minutes is found to be optimal time as for the longer period of placement can effect the results because of sweating or absorption of gel in skin. Further the hydrated and dehydrated data is collected in two postures named sitting and standing separately to study if the body posture has a considerable impact.

The overall length of the data collected is four hours approximately. Out of which data of two hours is for standing and two hour data is for sitting position approximately.FOr sitting one hour data is for dehydration state and almost one hour data is for hydration state. Same is the case for data of standing posture. For a window size of 30 seconds we get 120
TABLE I

Classification Results

\begin{tabular}{|l|l|l|l|l|l|}
\hline \multirow{2}{*}{ Model } & \multirow{2}{*}{ Data } & \multicolumn{4}{|c|}{ Accuracy for Window Sizes } \\
\cline { 3 - 6 } & & $\mathbf{3 0}(\mathbf{S e c})$ & $\mathbf{4 5}(\mathbf{S e c})$ & $\mathbf{6 0}(\mathbf{S e c})$ & $\mathbf{7 5}$ (Sec) \\
\hline \multirow{3}{*}{ KNN } & Sitting & $73.91 \%$ & $60.00 \%$ & $70.00 \%$ & $37.50 \%$ \\
\cline { 2 - 6 } & Standing & $81.82 \%$ & $73.33 \%$ & $60.00 \%$ & $37.50 \%$ \\
\cline { 2 - 6 } & Combined & $60.71 \%$ & $54.05 \%$ & $60.00 \%$ & $47.37 \%$ \\
\hline \multirow{3}{*}{ LR } & Sitting & $52.17 \%$ & $20.00 \%$ & $60.00 \%$ & $37.50 \%$ \\
\cline { 2 - 6 } & Standing & $50.00 \%$ & $53.33 \%$ & $40.00 \%$ & $12.50 \%$ \\
\cline { 2 - 6 } & Combined & $57.14 \%$ & $48.65 \%$ & $56.00 \%$ & $57.89 \%$ \\
\hline \multirow{3}{*}{ DT } & Sitting & $73.91 \%$ & $53.33 \%$ & $50.00 \%$ & $50.00 \%$ \\
\cline { 2 - 6 } & Standing & $59.09 \%$ & $80.00 \%$ & $70.00 \%$ & $50.00 \%$ \\
\cline { 2 - 6 } & Combined & $58.93 \%$ & $59.46 \%$ & $56.00 \%$ & $68.42 \%$ \\
\hline \multirow{3}{*}{ NVC } & Sitting & $73.91 \%$ & $60.00 \%$ & $70.00 \%$ & $37.50 \%$ \\
\cline { 2 - 6 } & Standing & $59.09 \%$ & $66.67 \%$ & $40.00 \%$ & $50.00 \%$ \\
\cline { 2 - 6 } & Combined & $57.14 \%$ & $56.76 \%$ & $64.00 \%$ & $57.89 \%$ \\
\hline \multirow{3}{*}{ LDA } & Sitting & $65.22 \%$ & $66.67 \%$ & $60.00 \%$ & $37.50 \%$ \\
\cline { 2 - 6 } & Standing & $59.09 \%$ & $46.67 \%$ & $60.00 \%$ & $50.00 \%$ \\
\cline { 2 - 6 } & Combined & $58.93 \%$ & $59.46 \%$ & $56.00 \%$ & $52.63 \%$ \\
\cline { 2 - 6 } & Sitting & $52.17 \%$ & $33.33 \%$ & $60.00 \%$ & $37.50 \%$ \\
\cline { 2 - 6 } & Combinged & $50.00 \%$ & $46.67 \%$ & $40.00 \%$ & $12.50 \%$ \\
\hline
\end{tabular}

segments of a data set from which we extracts 120 samples of each feature i.e there are 120 observations approximately, for each posture, sitting and standing, in each state, hydrated and dehydrated.

\section{Methodology}

Data Collection: Data is collected for a user in two different states called dehydrated, hydrated and two postures sitting and standing i.e. separate data is collected while sitting and standing before drinking water, remaining thirsty for longer hours, and same is repeated after drinking water.

Window Size: Set of window sizes used, to find optimal window of time, is $S=\{30,45,60,75\}$ where $s$ is the time in seconds for which the conductance data gathered is used to extract features.

Feature Extraction: The conductance data of each window of time is used to extract statistical features. Feature space created comprises the features set $F=$ mean, mod, median, variance . It is found that the combination of mean and variance of the conductance measured for given window size produced better results overall.

Machine Learning Models: In this research set of six classical machine learning classification algorithms are implemented. To name K-Nearest Neighbour (KNN), Logistic Regression(LR), Decsion Tree (DT), Support Vector Machine based Classifier (SVC), Gaussian Naive Byes Classifier (NB), Linear Discriminant Analysis (LDA), Decision Trees are used with different algorithm specific tuning parameters for example different values of $K$ the number of neighbour in KNN and different values of $C$ the regulator in SVC are tried.

Training, Testing and Optimization: $75 \%$ of the data is used to train and validate the models and $25 \%$ of the data is set separate to test each trained model. Overall eighteen separate models are trained, validated, tested and optimized using the above mentioned data sets, window sizes and machine learning classification algorithm with range of 
tuning parameters. Algorithms with respective data sets are listed in I. Three fold cross validation is used in validation step.

\section{Results}

It is found that even with the use of classical machine learning algorithm EDA can help to classify hydration level into dehydrated and hydrated level as shown in table I. For mean and variance used as features on a window of 30 seconds, KNN outperforms other algorithms for all three data sets, sitting,standing and combined. It reaches an accuracy of $81.82 \%$ for the sub data set for the standing posture. it provides an accuracy of $73.91 \%$ for the sitting data set and $60.71 \%$ for combined data set regardless of the posture. These results are obtained for the sets of tuning parameters \{metric: 'minkowski', $K$ : 19, weights: 'distance' $\},\{$ metric: 'minkowski', $K: 5$, weights: 'uniform'\} and \{metric: 'minkowski', $K: 20$, weights: 'uniform' $\}$ for sitting, standing and combined data sets respectively. It is also observed that with the parameters mentioned above KNN also performs good for class specific identification as shown figure 1. It exhibits an accuracy of $72.73 \%$ and $75 \%$ in identification of dehydration and hydration levels respectively for sitting data i.e it could identify $72 \%$ of the dehydration instances correctly and $75 \%$ of the hydration instances. Similarly, for standing data it identifies each of the hydration and dehydration instances with $82 \%$ accuracy, and for combined data with $38 \%$ and $78 \%$ accuracy for dehydration and hydration instances.

It is also observed that mostly algorithms perform better on the window size of 30 seconds particularly for posture specific data sets except few algorithm which performs better on window size of 45 seconds compared to their own performance on other windows e.g LR,DT, SVC for standing data,but their overall performance remains low. It is safe to say 30 second is an optimal window size for the feature extraction. A common behaviour is observed in all algorithms that they generally perform better on posture specific data as compared to posture independent data. It reflects that conductance not only varies because of hydration level but also the body posture. From this it can also be inferred that such data may also be helpful for identifying different body postures. From the results obtained so far we can safely say that, with the selection of appropriate machine learning algorithm and properly extracted features that overcome the phasic changes in EDA reading, EDA can be used for identifying hydration level in body. It is an abstract measure so far though.

\section{CONCLUSIONS}

Water is a vital component of life. In composition maximum volume and mass of human body is made of water. Decrease in water level also known as dehydration can effect the performance of human body and may lead to diseases in severe cases. It is, therefore, important to have efficient hydration monitoring solutions which are rare currently particularly non-invasive one. In this research we have proposed

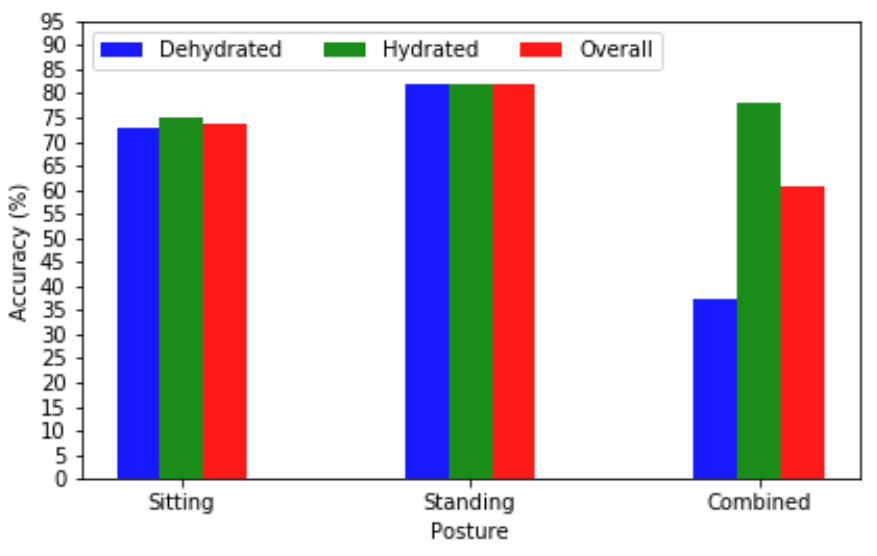

Fig. 1. Class specific performance of optimized KNN

a novel approach for the identification of hydration level. We have shown that EDA can be used to identify hydration level in human body in a non-invasive manner. With the help of KNN classification algorithm applied on EDA data collected from a human we could successfully classify hydration and dehydration level of human body with an accuracy upto $81.82 \%$ for posture specific data. This approach may be explored for further research on greater number of subjects and with advance feature extraction algorithms. It can also be extended to develop multi-class solution instead of binary class solution as it is the case here.

\section{ACKNOWLEDGMENT}

This work is funded by project AARE17-019 provided by the ADEC Award for Research Excellence, Abu Dhabi, United Arab Emirates Univesity.

\section{REFERENCES}

[1] Florian Lang and Siegfried Waldegger. Regulating cell volume. American scientist, 85(5):456, 1997.

[2] David Smith, Barbara Engel, Ann M Diskin, Patrik Španĕl, and Simon J Davies. Comparative measurements of total body water in healthy volunteers by online breath deuterium measurement and other near-subject methods. The American journal of clinical nutrition, 76(6):1295-1301, 2002.

[3] Lawrence E Armstrong. Assessing hydration status: the elusive gold standard. Journal of the American College of Nutrition, 26(sup5):575S584S, 2007.

[4] DA Schoeller, W Dietz, E Van Santen, and PD Klein. Validation of saliva sampling for total body water determination by $\mathrm{h} 218 \mathrm{o}$ dilution. The American journal of clinical nutrition, 35(3):591-594, 1982.

[5] Wm Cameron Chumlea and Shumei S Guo. Bioelectrical impedance and body composition: present status and future directions. Nutrition Reviews, 52(4):123-131, 1994.

[6] Raymond M Fish and Leslie A Geddes. Conduction of electrical current to and through the human body: a review. Eplasty, 9, 2009.

[7] Jason J Braithwaite, Derrick G Watson, Robert Jones, and Mickey Rowe. A guide for analysing electrodermal activity (eda) \& skin conductance responses (scrs) for psychological experiments. Psychophysiology, 49(1):1017-1034, 2013.

[8] BiTalino. Bitalino (r)evolution board kit data sheet.

[9] Society for Psychophysiological Research Ad Hoc Committee on Electrodermal Measures, Wolfram Boucsein, Don C Fowles, Sverre Grimnes, Gershon Ben-Shakhar, Walton T Roth, Michael E Dawson, and Diane L Filion. Publication recommendations for electrodermal measurements. Psychophysiology, 49(8):1017-1034, 2012. 